\title{
PPG prediction methodology by analyzing a simple lunch using GH-Method: math-physical medicine
}

\author{
Journal of Diabetes and Endocrinology Research
}

Short Communication

Gerald C. Hsu

EclaireMD Foundation, USA

\section{Introduction}

This paper discusses both predicted and measured postprandial plasma glucose (PPG) results from a simple lunch of one small bag of Quaker oatmeal: 18 grams carbs and 0 grams of sugar using the GH-Method: math-physical medicine (MPM). He developed MPM by applying mathematics, physics, engineering modeling, and computer science (big data analytics and AI). He believes in "prediction" and has developed five models, including metabolism index, weight, fasting plasma glucose (FPG), postprandial plasma glucose (PPG), and hemoglobin A1C. All prediction models have reached to $95 \%$ to $99 \%$ accuracy. His focus is on preventive medicine, especially on diabetes control via lifestyle management.

This article is written to aim at training dietitians, nurses, and other diabetes healthcare professionals.

\section{Methods}

The author applied signal processing of wave theory from earth physics and exploration along with electronics and communication engineering to decompose generalized PPG wave into 19 influential factors. This sophisticated physics analysis method has achieved a prediction accuracy of $>99.6 \%$ (based on $\sim 5,200$ meal data from the author).

He applied optical physics to develop an artificial intelligence AI-based Glucometer APP software on smartphones and PCs which includes only eight contribution factors, but also utilizes approximately eight million food nutritional data stored on a cloud server. This AI method's prediction accuracy has achieved $\sim 98 \%$ (based on a 3 to 12 -month meal data of seven patients).

Finally, in order to teach and train food nutritionists and dietitians in an easier way, he further simplified this nonlinear mathematical system and complex artificial intelligence model into a two-parameters based (food and exercise) linear equation

\author{
"Correspondence author \\ Gerald C Hsu, \\ EclaireMD Foundation \\ USA
}

Submitted : 02 June 2020 ; Published : 18 June 2020

to predict PPG. This linear equation model's prediction accuracy has achieved $\sim 95 \%$ (based on the same 3 to 12 -month meal data of 7 patients).

It is necessary to describe this two-parameter linear equation again (Reference no. 97):

Predicted $\mathrm{PPG}=(($ Baseline Glucose $\mathrm{A})+($ Carbs $/$ Sugar grams * Variable B) - (Walking Steps * Variable C) $) *$ Variable D

\section{Results}

The author's lunch on 09/07/2019 was a simple meal which contained a small bag of Quaker oatmeal (18 grams of carbs and 0 gram of sugar, see Figure 1).

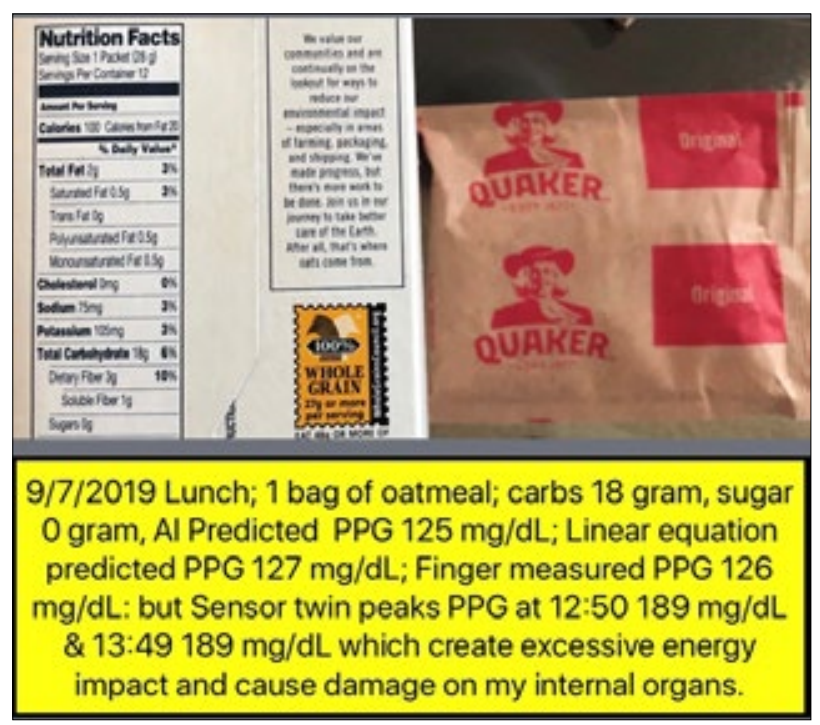

Figure 1: PPG results of a simple lunch - one packet of oatmeal 
He measured his PPG on the middle finger of his left hand, two hours after his first bite of lunch which resulted in a reading of $126 \mathrm{mg} / \mathrm{dL}$. His AI Glucometer APP predicted PPG was 125 $\mathrm{mg} / \mathrm{dL}$ and his two-parameters linear equation predicted PPG was $127 \mathrm{mg} / \mathrm{dL}$. Both prediction models have achieved the same $99.2 \%$ prediction accuracy for this particular meal.

At the same time, his seven major data for a total of 12 sensor collected PPG values (applied on his left arm) are listed below (see Figure 2):

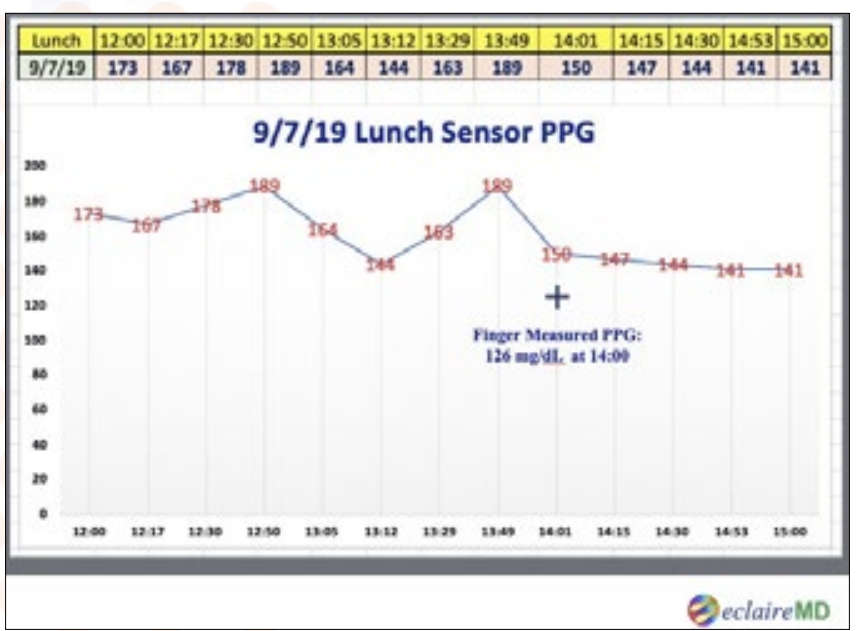

Figure 2: Sensor PPG record and graph

\begin{tabular}{|l|l|}
\hline Time (minutes) & PPG $(\mathrm{mg} / \mathrm{dL})$ \\
\hline 0 & 173 \\
\hline 15 & 167 \\
\hline 50 & 189 \\
\hline 72 & 144 \\
\hline 109 & 189 \\
\hline 121 & 150 \\
\hline 180 & 141 \\
\hline
\end{tabular}

This particular PPG waveform (i.e. curve) depicts a typical "twin peaks" pattern due to his higher carb intake $(25 \%$ higher than his averaged carbs intake at 14.4 grams per meal, see Figure 3) despite his post-meal walk of 6,000 steps. It should be noted that these twin peaks of $189 \mathrm{mg} / \mathrm{dL}$ are associated with high left-over energy which circulates within the blood vessels throughout the body and damaged his internal organs via CVD, stroke, renal complications, foot ulcer, bladder infection, eye problems, etc.

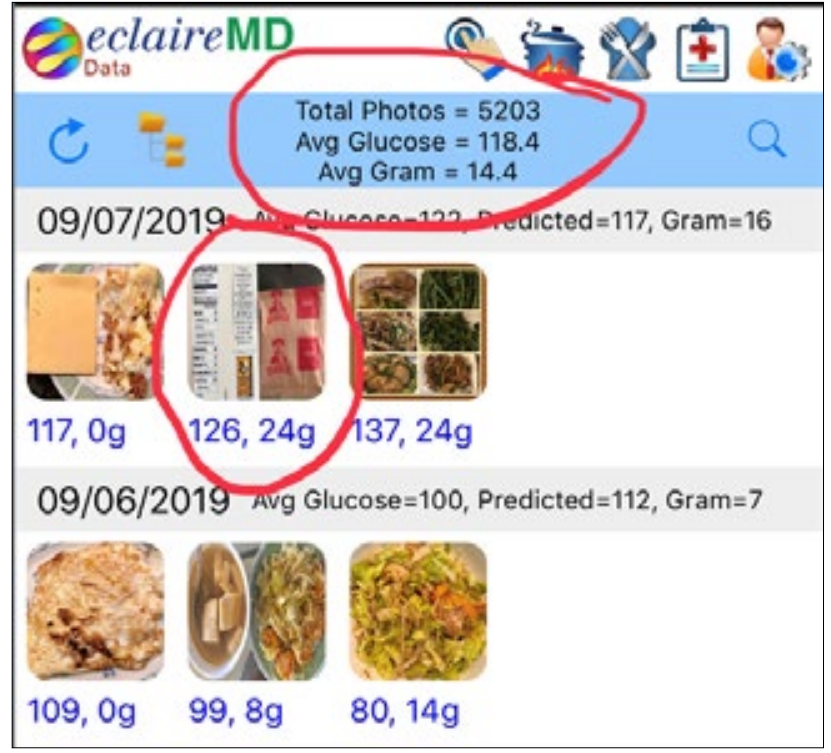

Figure 3: Author's meal record (a total of 5,203 meal photos and their average carbs/sugar 14.4 grams, average PPG 118.4 $\mathrm{mg} / \mathrm{dL}$ )

The author's baseline condition (see Reference no. 112, i.e. his pancreatic beta cell health state) is 121 . His carb intake would give him an increased glucose around $36-45 \mathrm{mg} / \mathrm{dL}$ and his 6,000 walking steps would give him a decreased glucose of approximately $30-45 \mathrm{mg} / \mathrm{dL}$. These two bands of glucose ranges are due to consideration of T2D severity in a particular patient. In this case, the linear equation operation is as follows:

\section{Predicted PPG \\ $=121+36-30$ \\ $=127 \mathrm{mg} / \mathrm{dL}$}

\section{Conclusion}

Although this article only describes a single meal's glucose situation, it is still useful through this example to explain the author's GH-Method: math-physical medicine (MPM) approach and its application on PPG prediction. There is no need to teach healthcare professionals this MPM approach in detail since it involves different background materials which takes years of learning. Once they can master the application of certain prediction techniques, they can help T2D patients to control their diabetes conditions effectively.

\section{References}

1. Hsu, Gerald C (2018) Using Math-Physical Medicine to Control T2D via Metabolism Monitoring and Glucose Predictions. Journal of Endocrinology and Diabetes 1(1): 1-6.

2. Hsu, Gerald C. (2018). Using Signal Processing Techniques to Predict PPG for T2D. International Journal of Diabetes \& Metabolic Disorders 3(2): 1-3.

3. Hsu, Gerald C (2018) Using Math-Physical Medicine and 
Artificial Intelligence Technology to Manage Lifestyle and Control Metabolic Conditions of T2D. International Journal of Diabetes \& Its Complications 2(3): 1-7.

4. Hsu, Gerald C (2018) Using Math-Physical Medicine to Study the Risk Probability of having a Heart Attack or Stroke Based on Three Approaches, Medical Conditions, Lifestyle Management Details, and Metabolic Index. EC Cardiology 5(12): 1-9.

Copyright: (C2020 Gerald C. Hsu. This is an open-access article distributed under the terms of the Creative Commons Attribution License, which permits unrestricted use, distribution, and reproduction in any medium, provided the original author and source are credited. 